\title{
The pathology of the lung in paraquat poisoning
}

\author{
PAUL SMITH AND DONALD HEATH \\ From the Department of Pathology, University of Liverpool
}

There have been numerous fatalities following accidental or deliberate ingestion of the weedkiller paraquat and these have received great attention both in the popular and scientific press. Most fatalities have occurred following the oral ingestion of Grammoxone, the concentrated form of the substance. Although this is not supposed to be readily available to the general public, people illicitly obtain samples of the weedkiller which they store at home in lemonade or beer bottles. In this way there is a great danger that the paraquat will be accidentally drunk by children or even unwary adults. Many fatalities have resulted from this careless disregard for precautions since only one mouthful of Grammoxone appears to be all that is required for a fatal outcome.

The disease is confined almost exclusively to the lungs and consists essentially of a fulminating pulmonary fibrosis which causes death from respiratory failure. The development of pulmonary lesions can be divided into two stages: a destructive phase and a proliferative phase.

\section{The Destructive Phase of Paraquat Poisoning}

The primary target for the destructive action of paraquat is the alveolar epithelium. In experimental rats there is a swelling of the type I alveolar epithelial cells or membranous pneumocytes only four hours after an intraperitoneal injection of paraquat (Smith and Heath, 1974a). By eight hours after injection membranous pneumocytes show vacuolation and disruption of organelles (Smith and Heath, 1974a) and by 18 hours these cells show hydropic degeneration in the form of numerous large vacuolated swellings projecting into the alveolus

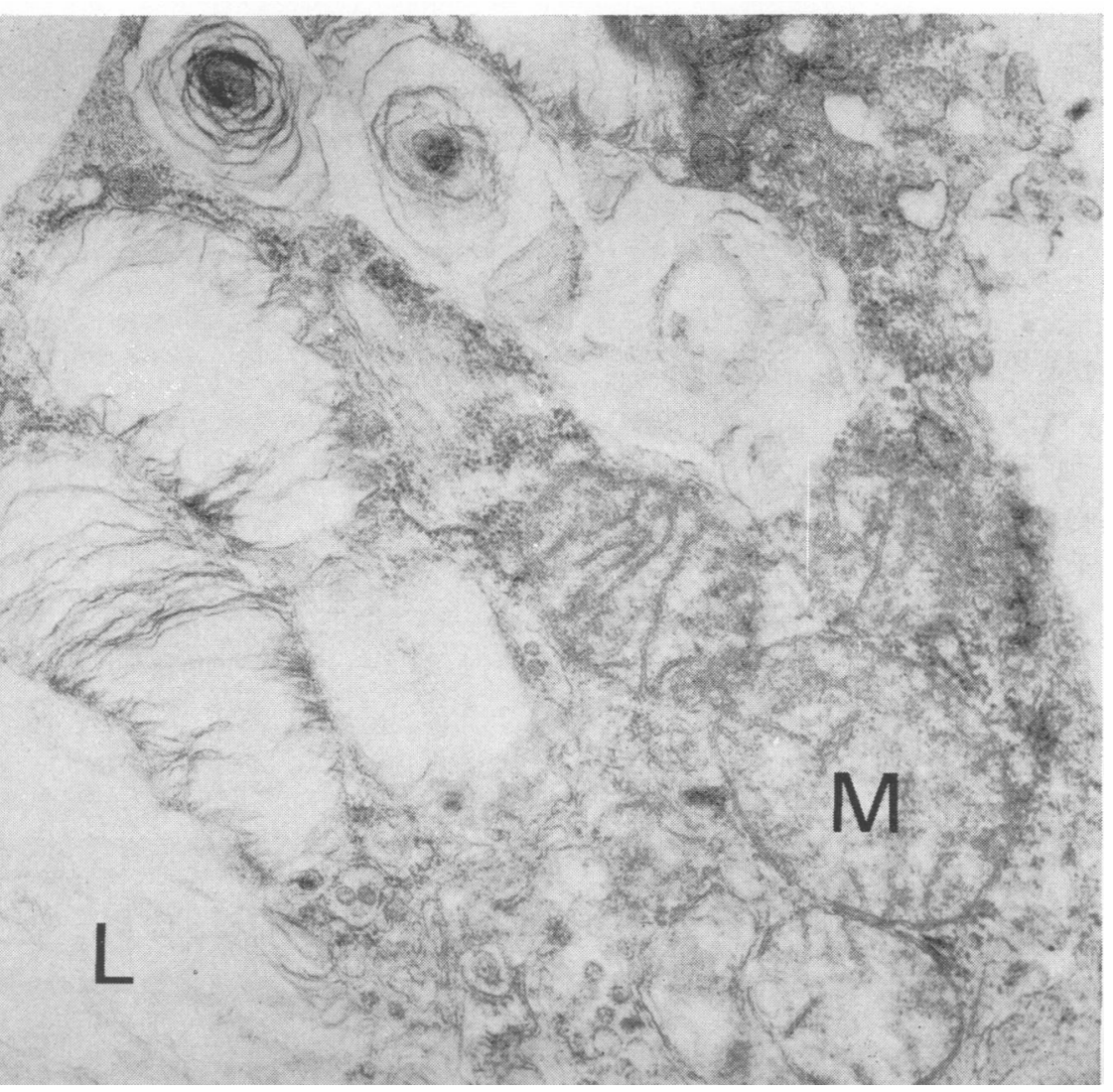

Fig 1 Granular pneumocyte from $a$ rat 24 hours after an injection of paraquat. The cell shows degenerative changes consisting of swelling of mitochondria $(M)$ and vacuolation of lamellar bodies $(L)$. There is also disruption of the rough endoplasmic reticulum. Electron micrograph $\times 25000$. 


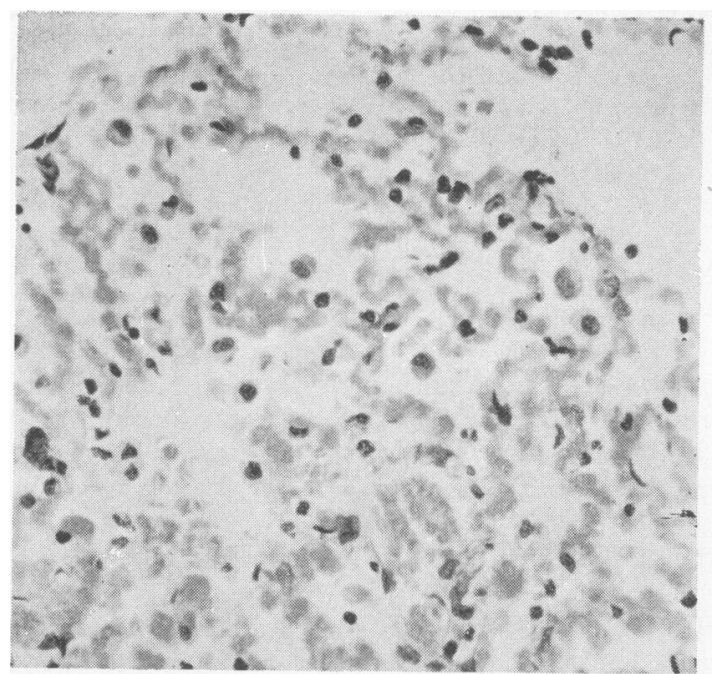

Fig 2 Three days after an injection of paraquat. Alveolar capillaries are congested with blood and there is alveolar pulmonary oedema. Neutrophil polymorphs are scattered about the lung. The intraalveolar mononuclear cells may be the first few profibroblasts to infiltrate the lung. Haematoxylin and eosin $\times 375$.

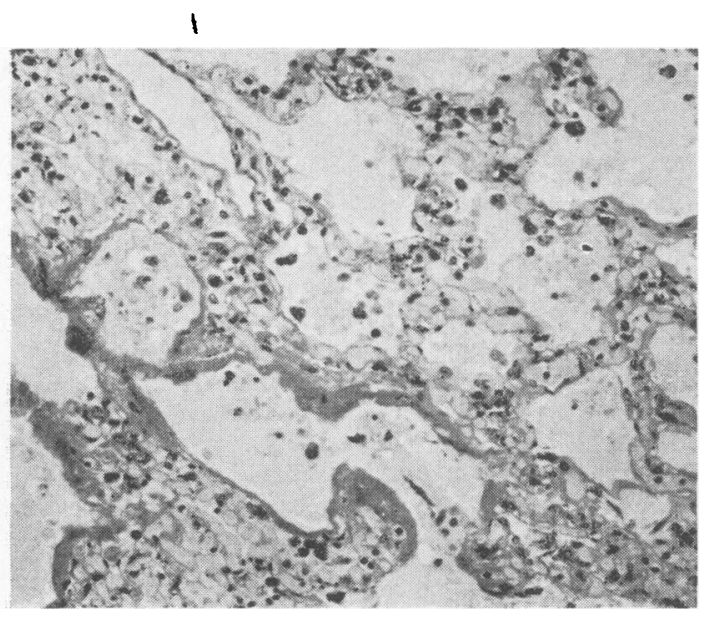

Fig 3 Human case of paraquat poisoning in which death from renal failure supervened while pulmonary lesions were at an early stage. The figure shows the destructive phase of paraquat lung in man comprising alveolar capillary congestion, alveolar oedema, fibrinous exudate, and neutrophil polymorphs. The respiratory bronchioles are lined by hyaline membranes. $H$ and $E \times 150$.
(Vijeyaratnam and Corrin, 1971; Smith and Heath, 1974a). Also at this time after injection degenerative changes occur in the type II alveolar epithelial cells or granular pneumocytes. These take the form of swelling of mitochondria, vacuolation of lamellar bodies and disruption of the endoplasmic reticulum (figure 1). Two days after the administration of paraquat both granular and membranous pneumocytes start to disintegrate so that by three days the alveolar walls are denuded of their epithelial lining (Smith and Heath, 1974a). It is interesting that this widespread destruction does not involve the alveolar capillaries.

In the rat, the sloughing of the alveolar epithelium is associated with alveolar pulmonary oedema, capillary congestion and a mild acute inflammatory reaction (figure 2). Eosinophilic hyaline membranes are also common (Manktelow, 1967; Robertson, Enhörning, Ivemark, Malmqvist, and Modée, 1971; Smith, Heath, and Kay, 1974). Alveolar pulmonary oedema during the early stage of paraquat poisoning in animals is often sufficiently extensive to cause severe dyspnoea, and many animals may die in this acute phase of the disease (Clark, McElligott, and Hurst, 1966; Smith et al, 1974).

In the destructive phase of paraquat poisoning in man, disintegration and sloughing of the alveolar epithelium also occur (Toner, Vetters, Spilg, and Harland, 1970). Although the sequence of changes leading to this lesion have not been determined it is probable that they are similar to those in the rat. Alveolar pulmonary oedema, capillary congestion, hyaline membranes and an acute inflammatory exudate are also found (figure 3). In man these changes are rarely fatal but may induce dyspnoea. Gardiner (1972) has demonstrated clinically the presence of pulmonary oedema during the early stages of paraquat poisoning.

The reason for the liberation of oedema fluid into the alveolar spaces is unclear since paraquat does not damage the pulmonary capillaries. Increased vascular permeability may account for the oedema (Vijeyaratnam and Corrin, 1971) but Gardiner (1972) has suggested that loss of pulmonary surfactant may be responsible. It is commonly accepted that the granular pneumocytes secrete pulmonary surfactant and, therefore, destruction of these cells by paraquat would lead to loss of surfactant with a corresponding increase in surface tension of the alveolar fluid. This could then withdraw fluid from the alveolar capillaries to produce oedema. Such an increase in surface tension has been demonstrated in animals (Manktelow, 1967; Robertson et al, 1971). These authors also suggest that loss of pulmonary surfactant leads to the formation of hyaline membranes, and for this reason they propose 
paraquat poisoning as an experimental model for the idiopathic respiratory distress syndrome of the newborn.

During the destructive phase of paraquat poisoning, damage to organs in the systemic circulation may occur. Thus, patients often show clinical evidence of hepatic and renal failure which usually resolves, after a few days (Almog and Tal, 1967; Campbell, 1968; Matthew, Logan, Woodruff, and Heard, 1968; Fennelly, Gallagher and Carroll, 1968). Occasionally renal failure may be the cause of death (Oreopoulos, Sayannwo, Sinniah, Fenton, McGeown, and Bruce, 1968; Smith and Heath, 1974b).

\section{The Proliferative Phase in Animals}

FOLLOWING A SINGLE DOSE OF PARAQUAT Approximately three days after a single injection of paraquat into rats scattered mononuclear cells are found in the alveolar spaces (figure 2), and by seven days alveolar spaces are filled with them (figure 4). These cells are irregular in shape and measure between 6 and $10 \mu \mathrm{m}$ in diameter. They present a 'ragged' outline, with non-vacuolated, moderately eosinophilic cytoplasm. Their nuclei are large, ovoid,

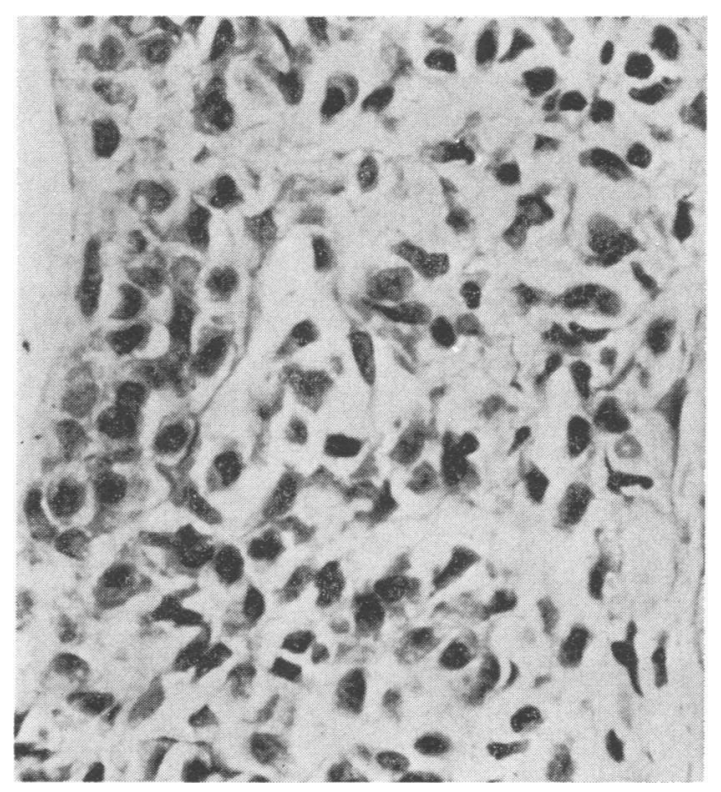

Fig 4 Rat seven days after an injection of paraquat. The lung is heavily infiltrated with profibroblasts which fill the alveolar spaces. Alveolar walls are disrupted. Note the irregular 'ragged' outline of the cells and their large, dark, eccentric nuclei. $H$ and $E \times 600$. darkly staining and eccentrically placed. Although these cells may superficially resemble macrophages they are not phagocytic but differentiate into fibroblasts. For this reason we have called them immature or profibroblasts (Smith et al, 1974).

The ultrastructure of a profibroblast is shown in figure 5. Their most prominent feature is the possession of numerous, long, filamentous pseudopodia at their periphery (figs 5 and 6). Their oval nuclei are smooth in outline with large quantities of dark chromatin. Mitochondria are small, rounded and dense with tightly packed cristae. At low magnifications mitochondria can be confused with the few small lysosomes also present in the cytoplasm (fig 5). Rough endoplasmic reticulum is scanty and consists of undulating parallel pairs of membranes bearing ribosomes. The pairs of membranes are unbranched and the spaces between them (cisternal spaces) are very narrow (fig 5). These profibroblasts are present exclusively within the alveolar spaces (fig 6) (Smith et al, 1974; Smith and Heath, 1974a).

Profibroblasts rapidly undergo differentiation into mature fibroblasts and the sequence of changes involved in this maturation has been traced in the rat (Smith et al, 1974; Smith and Heath, 1974a). Initially the cells become larger and more basophilic and then they elongate (fig 7). Mitotic figures are sometimes encountered. Later the cells undergo further elongation and increase in basophilia until they are mature fibroblasts. During this maturation the alveolar walls become disrupted (fig 7) until eventually they are unrecognizable. At an ultrastructural level the maturation of profibroblasts commences with the withdrawal of pseudopodia, enlargement of mitochondria and an increase in quantity of rough endoplasmic reticulum (fig 8). This is followed by branching of the rough endoplasmic reticulum and widening of cisternal spaces. Progressive elongation of these cells produces mature fibroblasts (fig 9). It has been demonstrated that these fibroblasts are present exclusively within the alveolar spaces (fig 10) and that alveolar walls are not infiltrated in any way (Smith et al, 1974). On the contrary alveolar walls are severely disrupted and often all that remains is the alveolar capillaries (figs 6 and 10). The histological appearance of the lung at this final stage of paraquat poisoning is that of a dense mass of fibroblastic tissue with almost total obliteration of the lung architecture (fig 11). Death usually supervenes about 10 days after injection before much collagen can be secreted. Electron microscopy shows small quantities of collagen contained within lacunae in the fibroblasts (fig 10). In this terminal stage of paraquat lung the origin of the pulmonary fibrosis is difficult to determine, so extensive is the obliteration of normal lung structure. 


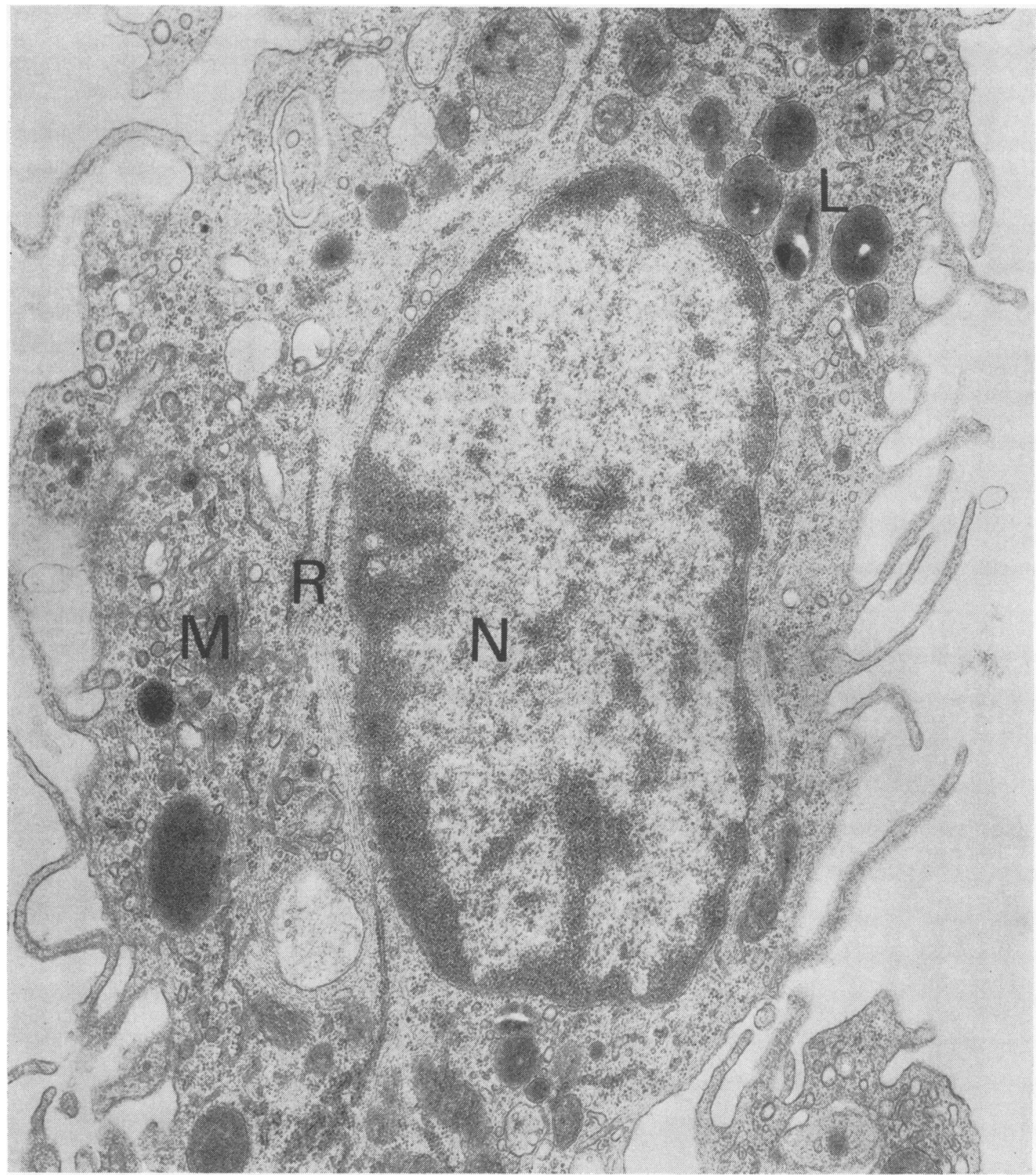

Fig 5 Electron micrograph from the same rat as in fig 4 showing details of a profibroblast. It has a large oval nucleus $(N)$ and the periphery is thrown into numerous filamentous pseudopodia. Mitochondria $(M)$ are inconspicuous and the rough endoplasmic reticulum $(R)$ consists of a few parallel membranes with narrow cisternae. A few lysosomes $(L)$ are also present. Electron micrograph $\times 18750$. 


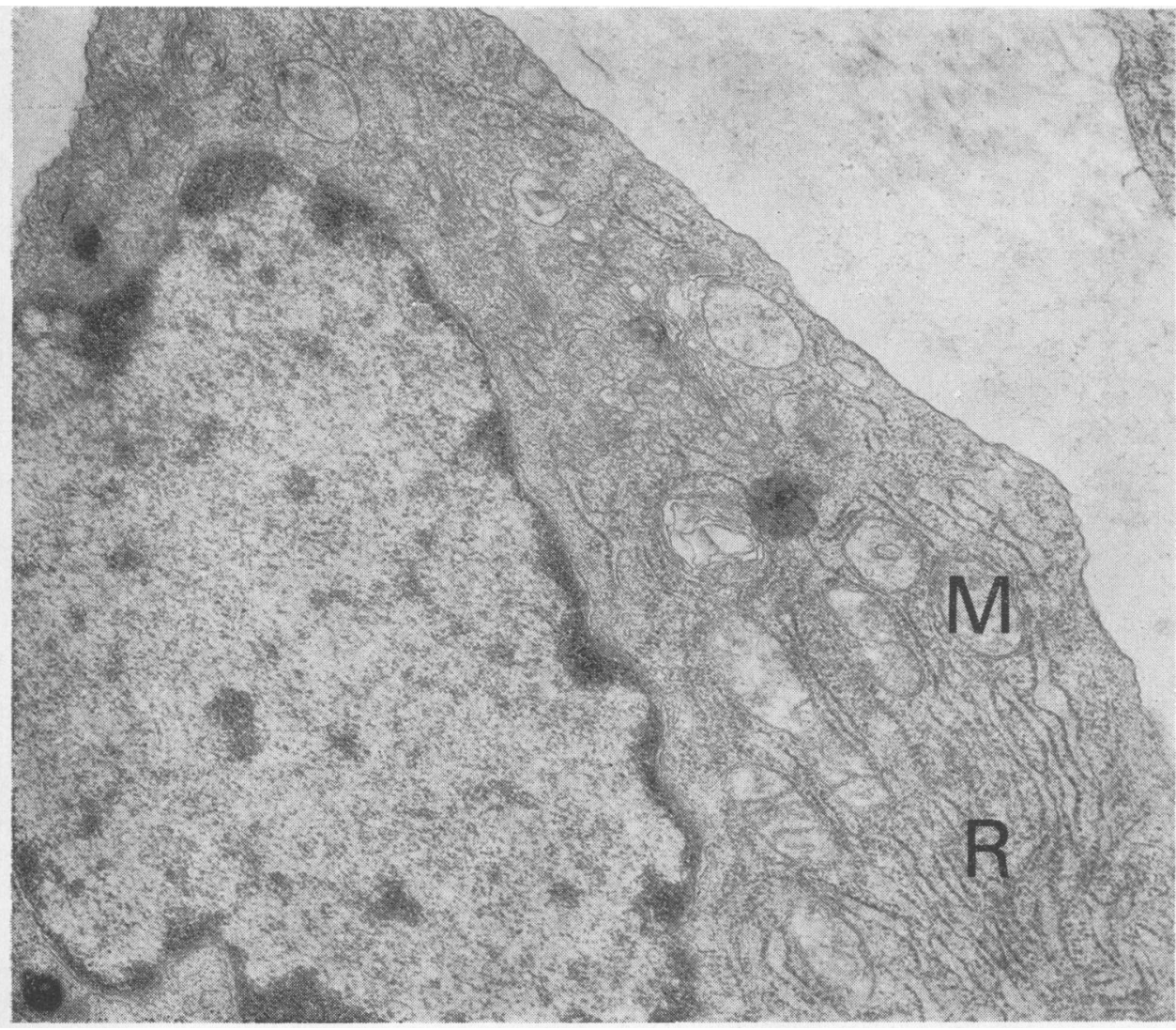

Fig 8 A cell intermediate in appearance between a profibrobla and a mature fibroblast. Note that it lacks pseudopodia. Mitochondria $(M)$ are larger and have a paler matrix than in figure 5. The rough endoplasmic $\overrightarrow{\vec{\omega}}$ reticulum $(R)$ is more extensive \& and cisternae are wider. Electrong micrograph $\times 25000$.

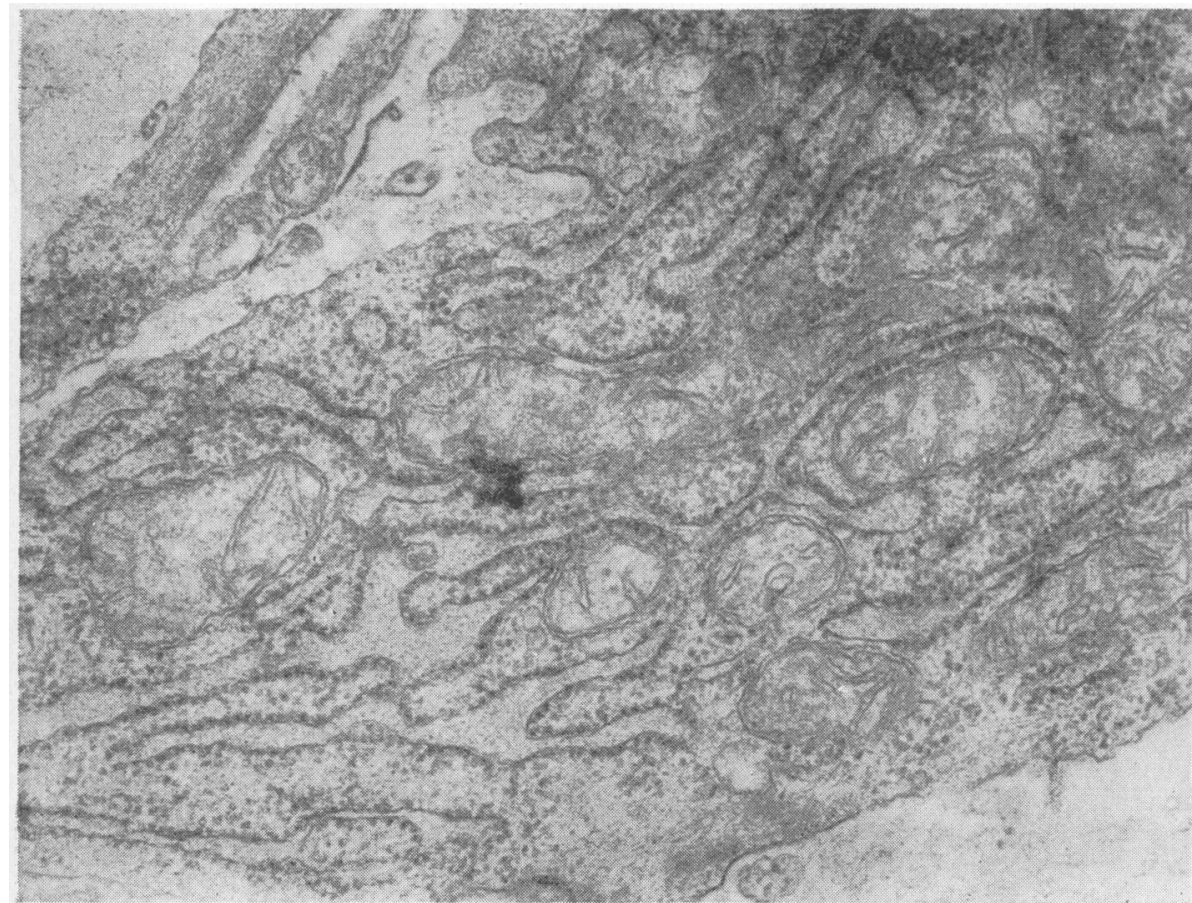

Fig 9 Part of a mature fibroblast from within an alveolar space. The cell is elongated, has prominent mitochondria 윽 and its rough endoplasmic reticulum is extensive with dilated cisternal spaces. Electron micrograph $\times 50000$. 


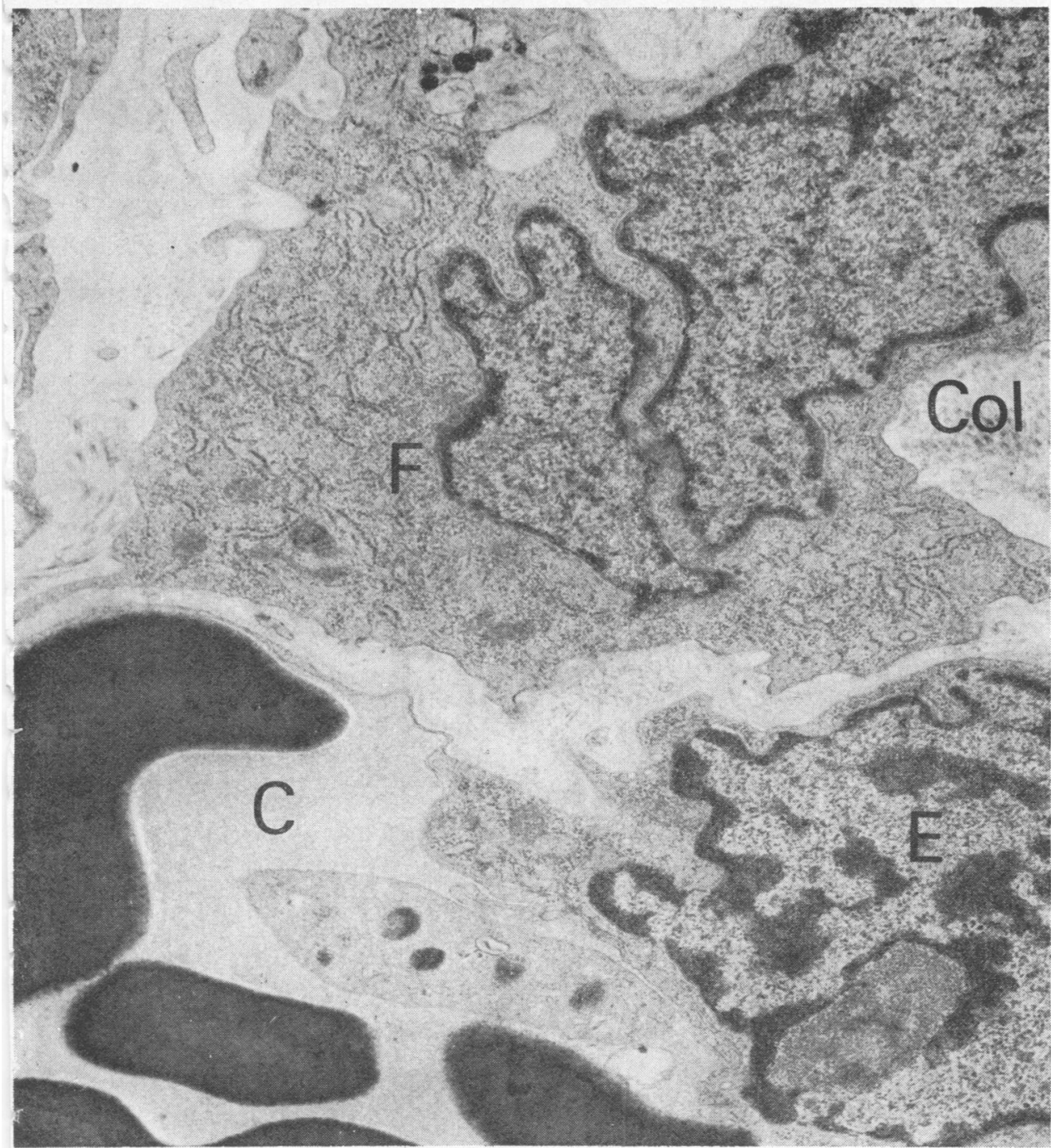

Fig 10

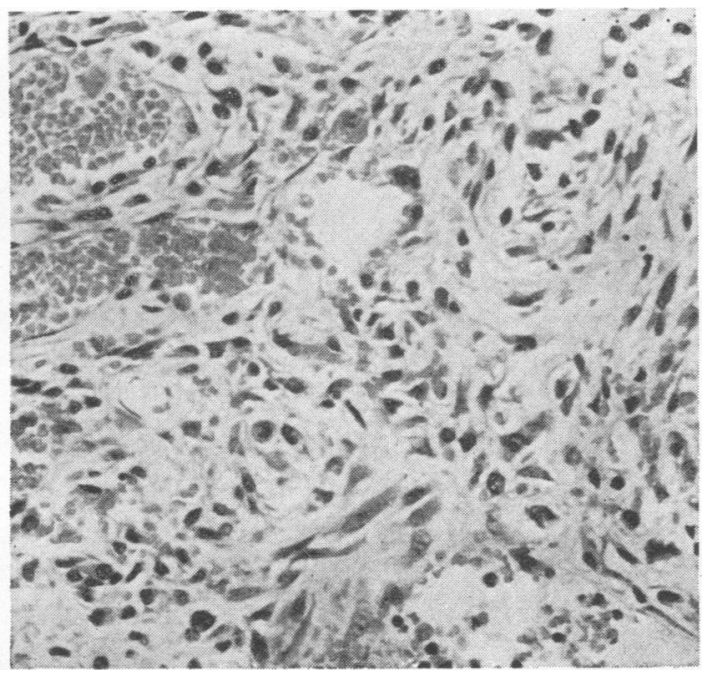

Fig 10 Lung of a rat 10 days after an injection of paraquat. A fibroblast $(F)$ can be seen within the alveolar space. The alveolar wall consists of capillary endothelial nucleus $(E)$ and capillary lumen $(C)$ containing erythrocytes and a platelet. The fibroblastic reaction is purely intraalveolar. Collagen fibrils $(\mathrm{Col})$ can be seen in the vicinity of the fibroblast. Electron micrograph $\times 18750$.

Fig 11 Lung of a rat 10 days after an injection of paraquat. The lung architecture is obliterated by a dense mass of fibroblasts and small quantities of collagen. $H$ and $E \times 375$.

Fig 11 
injection (Smith et al, 1974) or in the diet (Clark et al, 1966) pulmonary fibrosis results after a variable period of time. The pathogenesis of this fibrosis is the same as after a single dose (Smith et al, 1974) although the end result may be a looser fibrosis with fewer fibroblasts and more collagen (fig 12).

Occasionally one may encounter foci of interstitial fibrosis with patent alveoli containing foamy macrophages. There may be an associated hyperplasia of granular pneumocytes on the alveolar walls. It is unclear why this lesion should develop although an identical picture has been described in parts of the lungs of rats after prolonged intoxication with Crotalaria spectabilis seeds (Smith et al, 1970). Since these foci are uncommon it is unlikely that they play a significant role in paraquat lung. The proliferation of granular pneumocytes may represent areas of

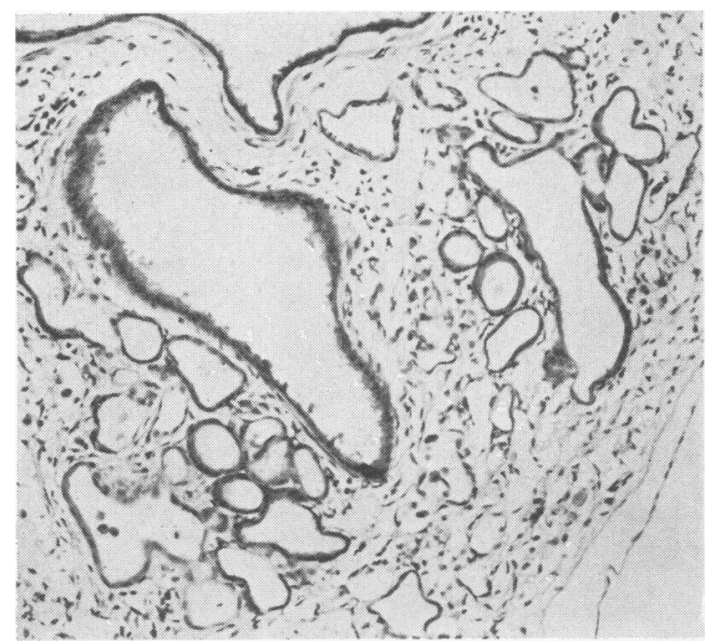

Fig 12 Rat following repeated injections of paraquat. There is a gland-like proliferation of the bronchiolar epithelium into surrounding alveolar ducts and alveoli. The fibrous tissue is much looser and more open in appearance than is seen after a single injection. $H$ and $E \times 150$.

epithelial regeneration where damage to the alveolar architecture has been less severe. We have never seen these changes in rats after single doses of paraquat.

Another lesion which is commonly found after repeated doses of paraquat is a proliferation of the bronchiolar epithelium (fig 12) into surrounding alveolar ducts and alveoli (Clark et al, 1966; Smith et al, 1974). This may also be an attempt at regeneration. The lesion is, however, a common finding in human necropsy cases where a single dose of paraquat has been taken.

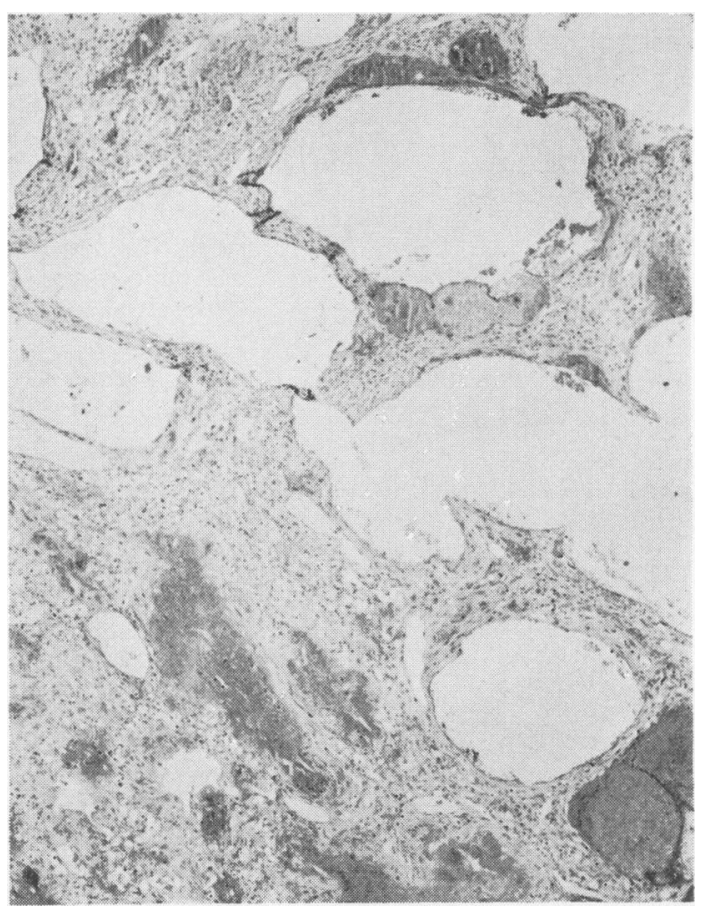

Fig 13 A human case of paraquat poisoning showing obliteration of the lung architecture by a dense mass of fibroblastic tissue. The large air spaces are dilated respiratory bronchioles which give the lung an early honeycomb appearance. Lakes of haemorrhage can also be seen. $H$ and $E \times 60$.

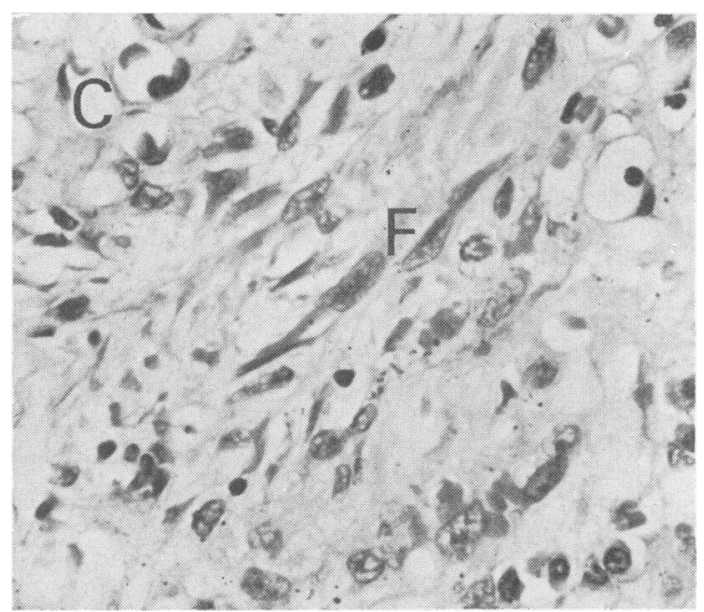

Fig 14 Detail of the fibroblastic tissue shown in figure 13. The fibroblasts $(F)$ are plump and basophilic and merge imperceptibly with fine collagen fibrils. Much of the intercellular matrix consists of fibrin and ground substance. The fibroblastic tissue includes numerous capillaries $(C)$. There is an infiltrate of lymphocytes and plasma cells $(H$ and $E \times 600)$. 


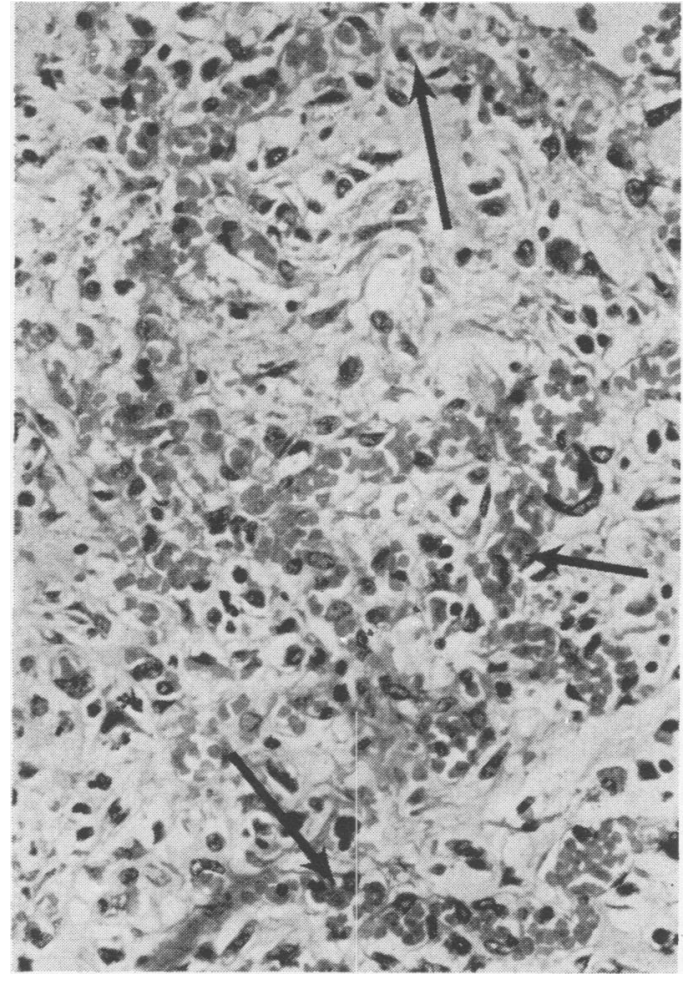

Fig 15 Human case of paraquat poisoning showing early intraalveolar fibrosis. Alveolar walls persist and can be seen as chains of capillaries (arrowed). The alveolar spaces contain fibroblasts but the alveolar walls are not infiltrated in any way. $H$ and $E \times 285$.

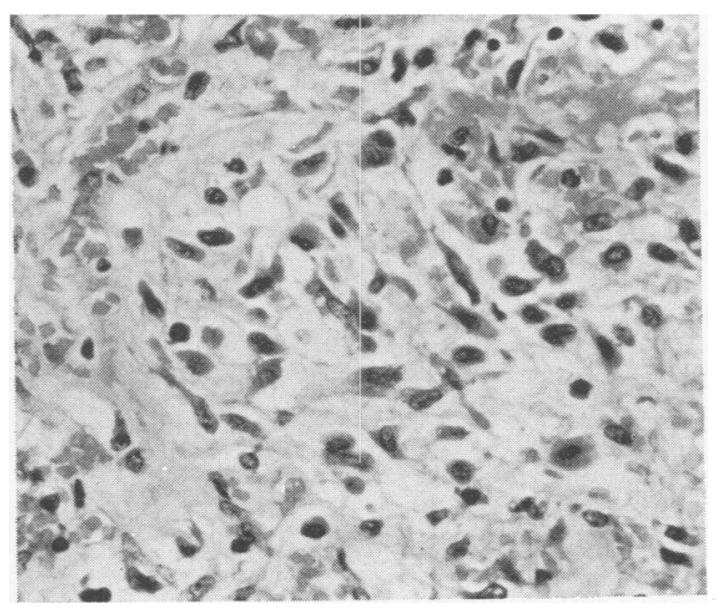

Fig 16 Same case as figure 15 showing a different area of lung. There is extensive intraalveolar fibrosis although the alveolar wall is more severely disrupted than in the previous figure. $H$ and $E \times 375$.

\section{The Proliferative Phase in Man}

Accidental or deliberate ingestion of paraquat by man commonly leads to the development of pulmonary fibrosis which causes death between one and three weeks after ingestion. Elucidation of the pathogenesis of this fibrosis is hampered by the fact that one is usually examining the advanced terminal stage of the disease. The histological picture typical of advanced paraquat lung in man is shown in figure 13. It consists essentially of a dense mass of fibroblastic tissue causing obliteration of the normal lung architecture. Surviving respiratory bronchioles may show dilatation to produce an early honeycomb appearance (fig 13) (Matthew et al, 1968; Smith and Heath, 1974b). In a few cases there is also pulmonary haemorrhage but this is not an invariable part of the histology. The fibroblastic tissue consists of numerous plump, basophilic fibroblasts with an acellular fibrillary matrix between them (fig 14). The matrix stains only faintly with Van Gieson's reagents and consists largely of immature collagen and fibrin. It is infiltrated by lymphocytes and plasma cells (fig 14) (Smith and Heath, 1974b). The pulmonary fibrosis also includes numerous capillaries (fig 14) which are presumably derived from alveolar capillaries following breakdown of the alveolar walls. In some cases, surviving alveoli may be lined by a continuous epithelium of granular pneumocytes (Matthew et al, 1968; Smith and Heath, 1974b) and there is commonly a gland-like proliferation of the bronchiolar epithelium.

This sort of histological picture in the lung in paraquat poisoning is often described as being an interstitial pulmonary fibrosis (Bullivant, 1966; Fennelly et al, 1968; Matthew et al, 1968), and paraquat lung is now held by many to be an example of diffuse fibrosing alveolitis as defined by Scadding and Hinson (1967). Recently we described a case of paraquat poisoning which suggested that this was not the case (Smith and Heath, 1974b). In this case the fibrosis was less dense and had caused less obliteration of the lung architecture than normal. Also, the alveolar walls, although damaged, had persisted and were easily identified as chains of capillaries containing red blood cells (fig 15). These acted as markers for the situation of the pulmonary fibrosis which was present exclusively in the alveolar spaces (fig 15). The fibrosis consisted of elongated, basophilic strap-shaped fibroblasts with long cytoplasmic prolongations which merged imperceptibly with ground substance and fine collagen fibrils (fig 16). A few cells were seen which were round or oval with an irregular 'ragged' outline, unvacuolated, moderately eosinophilic cytoplasm and large, darkly staining nuclei. These cells were similar in appearance 
to profibroblasts which have been described in connexion with paraquat-induced pulmonary fibrosis in rats.

We interpret these observations as representing an earlier stage of the proliferative phase of paraquat poisoning than one usually encounters. In this case the origin of the pulmonary fibrosis was demonstrated as being intraalveolar and not interstitial. We suggest that the pathogenesis of human paraquat lung may parallel that in the rat and consist of an intraalveolar infiltration of profibroblasts followed by their maturation into mature fibroblasts. Cells resembling profibroblasts were seen in the case just described and similar cells were observed in a human lung biopsy specimen (Toner et al, 1970), but were interpreted as a monocyte infiltration. At a subsequent necropsy of this case, these workers described an extensive pulmonary intraalveolar fibrosis. Thus the final stage of paraquat poisoning, like that in the rat, may be reminiscent of fibrosing alveolitis but obliteration of the alveolar walls belies its true intraalveolar origin. We think that the basic pathological change is a diffuse, cellular, intraalveolar fibrosis.

\section{The Influence of Oxygen Therapy}

The severe dyspnoea experienced by patients poisoned by paraquat requires treatment with artificial respiration of gas mixtures containing a high partial pressure of oxygen (Almog and Tal, 1967; Campbell, 1968; Oreopoulos et al, 1968; Matthew et al, 1968; Toner et al, 1970; Smith and Heath, 1974b). Prolonged respiration of gases containing more than $40 \%$ oxygen carries a grave risk of oxygen poisoning (Sevitt, 1974). In this condition there is an early exudative phase consisting of interstitial and alveolar oedema with the formation of eosinophilic hyaline membranes. This is followed by a proliferative phase after five days of oxygen therapy in which there is a hyperplasia of granular pneumocytes and the development of interstitial pulmonary fibrosis (Nash, Blennerhassett, and Pontoppidan, 1967; Sevitt, 1974). In some cases reviewed by Sevitt there was also an intraalveolar fibrosis. One should, therefore, consider the possibility that oxygen toxicity may be an important factor in the pathogenesis of pulmonary fibrosis in some cases of paraquat poisoning. It is clear that paraquat is capable of producing pulmonary damage on its own since intraalveolar fibrosis occurs when paraquat is given to rats breathing only atmospheric oxygen. Furthermore the histopathology of paraquat lung and oxygen poisoning is somewhat different in that, although intraalveolar fibrosis may occur in the latter, there is always a prominent interstitial component. Neither is there a profuse hyperplasia of granular pneumocytes in paraquat lung since these cells disintegrate and most alveolar walls are disrupted and become engulfed by fibroblastic tissue. One cannot, however, completely exclude oxygen toxicity as a contributory factor in the pathogenesis of pulmonary fibrosis in some cases of paraquat poisoning in man.

\section{The Mechanism of Toxicity of Paraquat}

Paraquat in its concentrated form (Grammoxone) has a strong irritant action on various types of epithelia. Thus it will cause blistering and soreness of the skin (Swan, 1969), damage to the corneal epithelium when splashed into the eyes (Swan, 1968), and damage to the nail-bed of the fingers causing shedding of the nails (Samman and Johnston 1969). Many patients following oral ingestion of Grammoxone show ulceration of the epithelium of the buccal cavity and fauces. It may be that a similar caustic action is responsible for the disintegration of the alveolar epithelium during the destructive phase of paraquat poisoning. However, when Grammoxone is diluted its dermal toxicity is very much reduced (Swan, 1969), and it seems unlikely that its concentration in the blood following ingestion would be high enough for the alveolar epithelium to be damaged merely by the caustic nature of the compound.

Paraquat appears to kill plants by entering the chloroplasts and then taking part in an oxidationreduction cycle in which hydrogen peroxide is liberated (Conning, Fletcher, and Swan, 1969). A similar type of calalytic activity may occur in animal tissues in which a small concentration of paraquat can lead to the synthesis of a high concentration of toxic byproducts. The highly vulnerable alveolar epithelium may succumb to these toxins, perhaps by inhibition of the sodium pump with consequent waterlogging of the cells.

Theories which may explain the destructive action of paraquat on the lung do not adequately explain the subsequent development of pulmonary fibrosis. Vijeyaratnam and Corrin (1971) suggested that this arose simply from organization of acute inflammatory exudate. However, chronic administration of paraquat to rats induces an extensive fibrosis following only a slight fibrinous exudate (Smith et al, 1974). Also, Gage (1968) had shown that inhalation of paraquat aerosols by animals causes an extensive exudate of oedema and fibrin into the alveoli but that pulmonary fibrosis never results from this. It seems that the formation of a fluid exudate during the destructive phase of paraquat poisoning 
is not necessary for the initiation of the proliferative phase which is independent of it.

In an attempt to explain the fibrogenic nature of paraquat, Conning et al (1969) studied organ cultures of lung. They found that paraquat causes extensive necrosis of alveolar cells but not of mesenchymal structures. Work with unicellular cultures showed that alveolar and peritoneal macrophages are more readily killed by paraquat than are fibroblasts. Furthermore, the addition of macrophages treated with paraquat to a culture of fibroblasts resulted in a more rapid proliferation of the latter. It appears therefore that paraquat actively stimulates proliferation of fibroblasts and that it achieves this through the medium of macrophages.

The fact that paraquat will only produce pulmonary fibrosis when administered systemically suggests that it is metabolized in another organ such as the liver and that a metabolite is at least partly responsible for the proliferative changes. This would certainly explain why pulmonary fibrosis in animals often develops after the paraquat concentration in the body has dropped below detectable levels (Daniel and Gage, 1966). In man there is often a small residue of paraquat in the body for up to 15 days after ingestion (Carson, 1972). What this metabolite may be, how it can stimulate an infiltration of profibroblasts into the lung, and why only the lung is affected, are all questions which cannot be answered in the present state of knowledge.

\section{Histopathology of the Pulmonary Vasculature in Paraquat Poisoning}

The pulmonary fibrosis in paraquat poisoning is frequently associated with pulmonary vascular lesions. The type of change in the pulmonary blood vessels seems to depend upon the degree of honeycomb change in the lung (Smith and Heath, 1974b).

Where there is a dense, cellular, obliterative pulmonary fibrosis with little dilatation of respiratory bronchioles, the muscular pulmonary arteries show a moderate degree of medial hypertrophy (fig 17). There is also a pronounced muscularization of pulmonary arterioles. In both these classes of vessel there is a cellular intimal proliferation consisting of fine elastic fibres and longitudinally orientated smooth muscle (fig 17). A minority of vessels may show focal atrophy of the media with splitting of the external elastic laminae. A slight degree of medial hypertrophy of muscular pulmonary arteries, muscularization of arterioles and longtitudinal muscle in the intima have all been described in cases of hypertensive pulmonary vascular disease (HPVD) brought about by conditions of chronic hypoxia (Hasleton, Heath, and Brewer, 1968). Thus it is

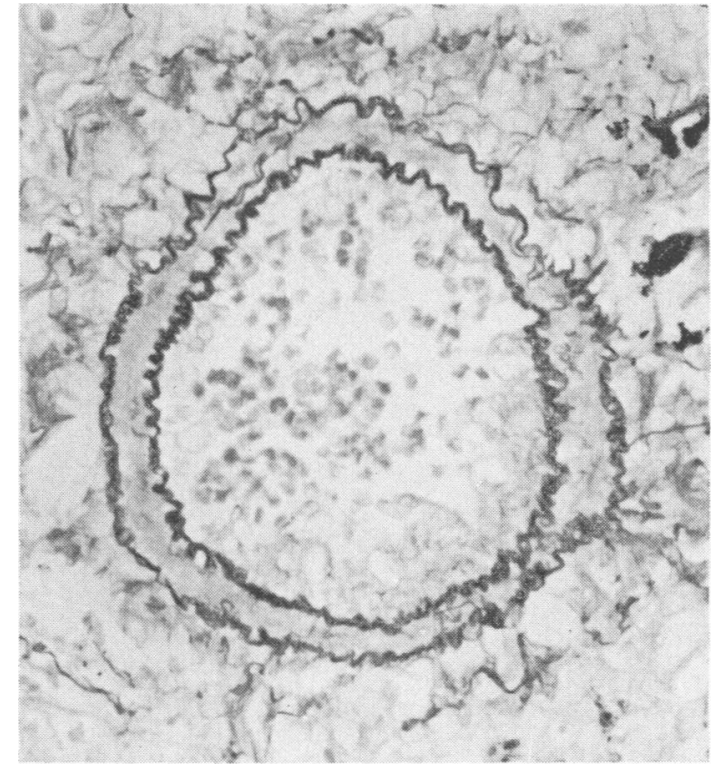

Fig 17 Human case of paraquat poisoning showing a muscular pulmonary artery with hypertrophy of its media and an intimal proliferation of longitudinally orientated smooth muscle cells and fine elastic fibrils. Elastic Van Gieson × 375 .

possible that pulmonary fibrosis in paraquat poisoning produces alveolar hypoxia of sufficient severity to induce the changes of hypoxic hypertensive pulmonary vascular disease, despite the fact that death occurs a mere three weeks after ingestion.

Where there has been extensive honeycomb change, the pulmonary vascular pathology is different. In the larger muscular pulmonary arteries the media is atrophic so that individual smooth muscle cells are widely separated (fig 18). External elastic laminae commonly show splitting and fragmentation. The intimal proliferation in these vessels is acellular consisting of anastomosing elastic fibres with fine collagen fibres in its interstices (fig 18). Longitudinally orientated smooth muscle cells are rare or absent. The medias of smaller muscular pulmonary arteries are distinctly atrophic and fragmentation of both elastic laminae is extensive (fig 19). In some pulmonary arteries medial atrophy and fragmentation of elastic laminae is so severe that they consist of an irregular ring of fibroelastic tissue with a diminished lumen. Small pulmonary veins contain an extensive intimal fibrosis of fine collagen fibrils which may totally occlude the affected vessel. Similar changes have been described in the pulmonary arteries of honeycomb lung complicating a variety of diseases (Heath, Gillund, Kay, and Hawkins, 1968). 


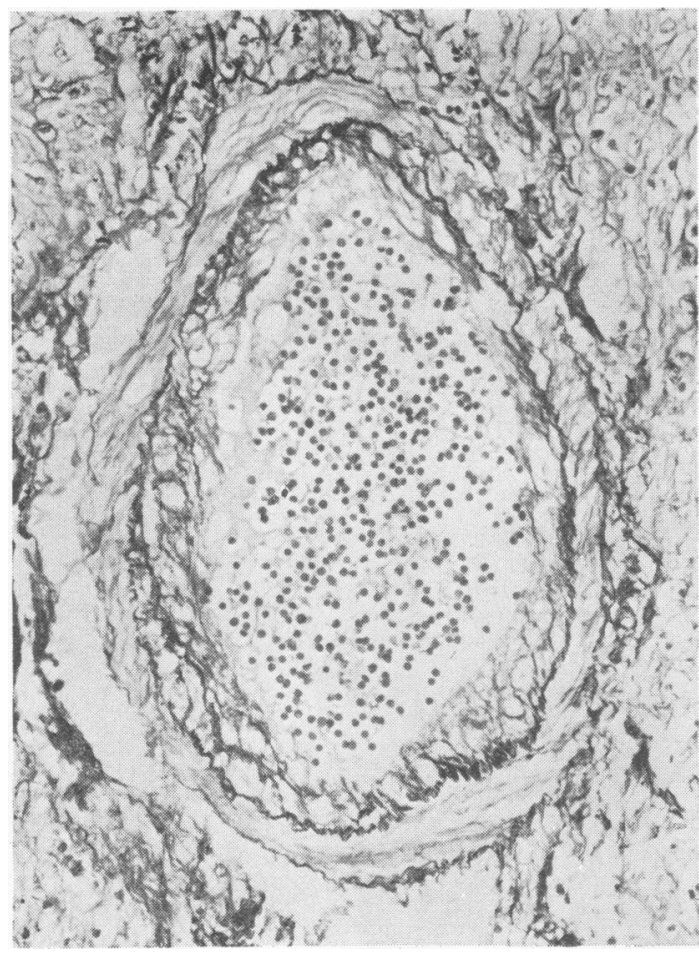

Fig 18

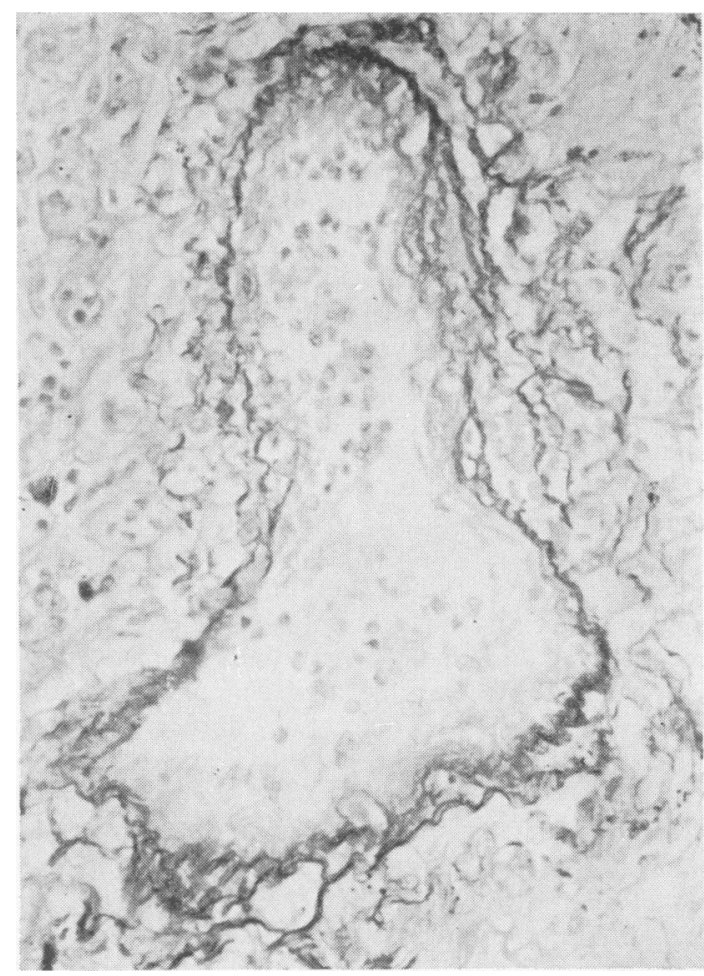

Fig 19

Fig 18 Large muscular pulmonary artery showing atrophy of the smooth muscle cells in its media. There is an intimal proliferation consisting of elastic fibres and fine collagen fibres. EVG $\times 232$.

Fig 19 Muscular pulmonary artery showing extensive medial atrophy and fragmentation of both elastic laminae. $E V G \times 375$.

\section{Conclusions}

The pathogenesis of paraquat lung involves a destructive phase and a proliferative phase, and these two stages appear to be independent of one another. The destructive phase consists of swelling and fragmentation of the alveolar epithelium followed by alveolar oedema and an acute inflammatory exudate. In rats the proliferative phase commences with an infiltration into the alveolar spaces of profibroblasts which then mature via a series of stages into mature fibroblasts to produce a diffuse intraalveolar fibrosis. There is evidence that the proliferative phase in man has a similar pathogenesis. The pulmonary fibrosis in human paraquat lung is not therefore a type of fibrosing alveolitis, but is instead a diffuse, cellular intraalveolar fibrosis.

Although various theories have been postulated to explain the destructive action of paraquat in the lung, there is no satisfactory explanation for the development of pulmonary fibrosis. This has made treatment of the condition extremely difficult. The cellular nature of the fibrosis plus its rapid development probably explains the lack of success of steroid therapy and this treatment is no longer recommended. A degree of success has been claimed from the combined use of steroid and cyclophosphamide (Lancet, 1971). Treatment usually consists of attempting to remove the paraquat from the body before the damage is done. Thus emetics, forced diuresis, and haemodialysis are common forms of treatment. Because paraquat is adsorbed on to fine particles, kaolin or fuller's earth is sometimes administered. However, once the fibrotic changes are initiated, they progress inexorably and there is little that clinicians can do about it. Until the mechanism of paraquat toxicity is more fully understood we can only search blindly for an antidote. In the meantime the onus is on the general public not to decant Grammoxone from the containers in which it is supplied and thereby reduce the chances of accidental ingestion of the compound. 


\section{References}

Almog, C., and Tal, E. (1967). Death from paraquat after subcutaneous injection. Brit. med. J., 3, 721.

Bullivant, C. M. (1966). Accidental poisoning by paraquat: report of two cases in man. Brit. med. J., 1, 1272-1273.

Campbell, S. (1968). Death from paraquat in a child. (Letter.) Lancet, $1,144$.

Carson, E. D. (1972). Fatal paraquat poisoning in Northern Ireland. J. forens. Sci. Soc., 12, 437-443.

Clark, D. G., McElligott, T. F., and Hurst, E. W. (1966). The toxicity of paraquat. Brit. J. ind. Med., 23, 126-132.

Conning, D. M., Fletcher, K., and Swan, A. A. B. (1969). Paraquat and related bipyridyls. Brit. med. Bull., 25, 245-249.

Daniel, J. W., and Gage, J. C. (1966). Absorption and excretion of diquat and paraquat in rats. Brit. J. ind. Med., 23, 133-136.

Fennelly, J. J., Gallagher, J. T., and Carroll, R. J. (1968). Paraquat poisoning in a pregnant woman. Brit. med. J., 3, 722-723.

Gage, J. C. (1968). Toxicity of paraquat and diquat aerosols generated by a size-selective cyclone: effect of particle size distribution. Brit. J. ind. Med., 25, 304-314.

Gardiner, A. J. S. (1972). Pulmonary oedema in paraquat poisoning. Thorax, 27, 132-135.

Hasleton, P. S., Heath, D., and Brewer, D. B. (1968). Hypertensive pulmonary vascular disease in states of chronic hypoxia. $J$. Path. Bact., 95, 431-440.

Heath, D., Gillund, T. D., Kay, J. M., and Hawkins, C. F. (1968). Pulmonary vascular disease in honeycomb lung. J. Path. Bact., 95, 423-430.

Lancet (1971) Editorial. Paraquat poisoning. Lancet, 2, 1018-1019.

McCann, B. G., and Brewer, D. B. (1974). A case of desquamative interstitial pneumonia progressing to 'honeycomb lung'. J. Path., 112, 199-202.

Manktelow, B. W. (1967). The loss of pulmonary surfactant in paraquat poisoning: a model for the study of the respiratory distress syndrome. Brit. J. exp. Path., 48, 366-369.

Matthew, H., Logan, A., Woodruff, M. F. A., and Heard, B. (1968). Paraquat poisoning-lung transplantation. Brit. med. J., 3, 759-763.
Nash, G., Blennerhassett, J. B., and Pontoppidan, H. (1967). Pulmonary lesions associated with oxygen therapy and artificial ventilation. New Engl. J. Med., 276, 368-374.

Oreopoulos, D. G., Soyannwo, M. A. O., Sinniah, R., Fenton, S. S. A., McGeown, M. G., and Bruce, J. H. (1968). Acute renal failure in case of paraquat poisoning. Brit. med. J., 1, 749-750.

Robertson, B., Enhörning, G., Ivemark, B., Malmqvist, E., and Modée, J. (1971). Experimental respiratory distress induced by paraquat. J. Path., 103, 239-244.

Samman, P. D., and Johnston, E. N. M. (1969). Nail damage associated with handling of paraquat and diquat. Brit. med. J., 1, 818-819.

Scadding, J. G., and Hinson, K. F. W. (1967). Diffuse fibrosing alveolitis (diffuse interstitial fibrosis of the lungs). Thorax, 22, 291-304.

Sevitt, S. (1974). Diffuse and focal oxygen pneumonitis. J. clin. Path., 27, 21-30.

Smith, P., and Heath, D. (1974a). The ultrastructure and time sequence of the early stages of paraquat lung in rats. J. Path., $114,177-184$

Smith, P., and Heath, D. (1974b). Paraquat lung: a reappraisal. Thorax, 29, 643-653.

Smith, P., Heath, D., and Kay, J. M. (1974). The pathogenesis and structure of paraquat-induced pulmonary fibrosis in rats. J. Path., 114, 57-67.

Smith, P., Kay, J. M., and Heath, D. (1970). Hypertensive pulmonary vascular disease in rats after prolonged feeding with Crotalaria spectabilis seeds. J. Path., 102, 97-106.

Swan, A. A. B. (1968). Ocular damage due to paraquat and diquat. (Letter). Brit. med. J., 2, 624.

Swan, A. A. B. (1969). Exposure of spray operators to paraquat. Brit. J. ind. Med., 26, 322-329.

Toner, P. G., Vetters, J. M., Spilg, W. G. S., and Harland, W. A. (1970). Fine structure of the lung lesion in a case of paraquat poisoning. J. Path., 102, 182-185.

Vijeyaratnam, G. S., and Corrin, B. (1971). Experimental paraquat poisoning: a histological and electron-optical study of the changes in the lung. J. Path., 103, 123-129. 